\title{
Bladder attack: transient bladder ischemia leads to a reversible decrease in detrusor compliance
}

\author{
Andrew T. Tracey ${ }^{1 \#}$, Uzoma A. Anele ${ }^{1 \#}$, Randy A. Vince ${ }^{1}$, John E. Speich ${ }^{2}$, Adam P. Klausner ${ }^{1}$, Paul H. Ratz ${ }^{3}$ \\ ${ }^{1}$ Division of Urology, Department of Surgery, ${ }^{2}$ Department of Mechanical and Nuclear Engineering, ${ }^{3}$ Department of Biochemistry and Molecular \\ Biology, Virginia Commonwealth University School of Medicine, Richmond, VA, USA \\ Contributions: (I) Conception and design: All authors; (II) Administrative support: All authors; (III) Provision of study materials or patients: AT \\ Tracey, UA Anele, RA Vince, AP Klausner; (IV) Collection and assembly of data: AT Tracey, UA Anele; (V) Data analysis and interpretation: All \\ authors; (VI) Manuscript writing: All authors; (VII) Final approval of manuscript: All authors. \\ \#These authors contributed equally to this work. \\ Correspondence to: Adam P. Klausner, MD. Division of Urology, Department of Surgery, Virginia Commonwealth University School of Medicine, PO \\ Box 980118, Richmond, VA 23298-0118, USA. Email: adam.klausner@vcuhealth.org.
}

Background: The deleterious effects of chronic ischemia on bladder function have been extensively studied; however, evaluation and characterization of the effects of acute ischemia and hypoxia are lacking. The present study examined pig and human detrusor smooth muscle (DSM) strips, in combination with an isolated perfused working pig bladder model to evaluate the relationship between transient ischemia and bladder function.

Methods: Organ bath and myographic studies were performed using pig and human DSM strips exposed to starvation/hypoxia conditions. Analogous conditions were then recreated in the ex vivo bladder preparation. Filled bladders were then treated with intravascular carbachol to induce contraction and subsequent void. An intravesical transducer continuously monitored changes in bladder pressure, while a tissue $\mathrm{pO}_{2}$ monitor analyzed changes in oxygenation.

Results: After $120 \mathrm{~min}$ in starved/hypoxic conditions, both pig and human DSM strips demonstrated significantly increased resting tone, with a greater than two-fold increase in force over control. This was effectively blocked with atropine. DSM strips also demonstrated significantly weaker contractions; however, contractile force was nearly recovered following 15-min exposure to replete/oxygenated buffer. In the ex vivo bladder preparation, filling under ischemic conditions yielded a $225 \%$ increase in end-fill vesical pressures $\left(\mathrm{P}_{\text {ves }}\right)$ compared to controls. End-fill $\mathrm{P}_{\text {ves }}$ returned to baseline with reperfusion during a subsequent filling cycle.

Conclusions: Transient ischemia/hypoxia leads to an acute increase in tone in both DSM strips and ex vivo pig bladder. Remarkably, the effect is reversible with re-perfusion and may be blocked with anticholinergics, suggesting a relationship between acute ischemia and increased local acetylcholine release.

Keywords: Smooth muscle; hypoxia; reperfusion; myography; pathophysiology

Submitted May 27, 2019. Accepted for publication Nov 05, 2019.

doi: $10.21037 /$ tau.2019.11.12

View this article at: http://dx.doi.org/10.21037/tau.2019.11.12

\section{Introduction}

The clinical phenomena of overactive and underactive bladder dysfunction present a challenge for both patients and clinicians; however, the underlying pathophysiology is not well-understood. During normal filling or increased pressure, perfusion to the bladder decreases yet normal functionality is preserved. In the setting of chronic ischemia, the mechanisms that maintain this functionality may potentially be overcome, leading to aberrations which 
progress over time from bladder overactivity to eventual underactivity $(1,2)$. Current evidence suggests that pelvic ischemia plays a significant role in this pathway (3). Ischemic changes have been associated with worsening severity of lower urinary tract symptoms in the elderly patient population among whom these vasculopathies are common. Furthermore, population-based studies have also suggested that peripheral vascular disease may play a role in bladder dysfunction (4).

Experimental animal models of chronic ischemia have delineated a progression to detrusor overactivity, and eventually detrusor underactivity (5). The progression of these changes has been attributed to bladder noncompliance consequent to fibrosis (6). However, the acute changes in detrusor function in response to transient ischemia have not been fully elucidated. Therefore, we sought to evaluate the effect of acute ischemia on both the filling/storage and voiding phases of the bladder using pig and human detrusor smooth muscle (DSM) and an ex vivo pig bladder preparation.

\section{Methods}

All experiments involving human tissue received approval from the Virginia Commonwealth University institutional review board (HM 20009688) and informed consent was obtained from patients prior to their use. Porcine tissue experiments performed in this study did not require approval by the institutional animal care and use committee because they were acquired from abattoirs and not from laboratory animals.

\section{Pig bladder procurement}

Whole pig bladders were harvested en bloc from freshly slaughtered pigs at local abattoirs. The vesical arteries were immediately perfused with a cold heparinized KrebsHenseleit buffer (KHB) until the effluent was noted to be clear as previously described (7). The KHB composition was (in $\mathrm{mM}$ ): $\mathrm{NaCl}$ (114.0), $\mathrm{KCl}$ (4.7), $\mathrm{CaCl}_{2}$ (2.5), $\mathrm{MgSO}_{4}(1.2)$, $\mathrm{CaCl}_{2}$ dihydrate (2.5), $\mathrm{MgSO}_{4}$ monohydrate (1.2), $\mathrm{KH}_{2} \mathrm{PO}_{4}$ (1.2), $\mathrm{NaHCO}_{3}$ (25.0), D-glucose (11.7) and ascorbic acid (1.1), and the $\mathrm{pH}$ was adjusted to 7.4. Bladders were then transported to the laboratory in cold buffer for use within $24 \mathrm{~h}$.

\section{DSM tissue strip preparation}

Following procurement, portions of detrusor muscle were dissected from the anterior bladder wall. Pig DSM strips were prepared according to an established, standardized protocol (8). The tissue was then washed with KHB solution, denuded, and stored in cold $\left(0-4{ }^{\circ} \mathrm{C}\right)$ buffered physiologic saline solution. Thin longitudinal strips (approximately $1 \mathrm{~mm}$ thickness, $3 \mathrm{~mm}$ width, $15 \mathrm{~mm}$ length) were then created for placement in organ baths. They were attached by small clips to a micrometer and force transducer (159901a, Radnoti Glass Technology, Inc, Monrovia, CA, USA), submerged in a $30 \mathrm{~mL}$ water-jacketed, tissue bath containing aerated $\left(95 \% / 5 \% \mathrm{O}_{2} / \mathrm{CO}_{2}\right.$ gas) $\mathrm{KHB}$ at a slack length of $5 \mathrm{~mm}$, and allowed to equilibrate at $37^{\circ} \mathrm{C}$ as previously described $(8,9)$.

\section{Determination of reference tension and length for DSM strips}

DSM strips were suspended without any visible tension and a force of $<0.05 \mathrm{~g}$, and left to equilibrate for a minimum of 60 minutes. Isometric tension was measured at $10 \mathrm{~Hz}$ and voltage signals were then digitized (model DIO-DAS16, ComputerBoards, Mansfield, MA, USA) and analyzed using a multichannel data integration program (DASYLab 10.0, DasyTec, Amherst, NH, USA). After the tissues were equilibrated, an abbreviated length-tension protocol was used to determine a reference muscle length $\left(\mathrm{L}_{\mathrm{ref}}\right)$ (9). This validated stair-step length-tension protocol was applied to each DSM strip in $2 \mathrm{~mm}$ increments to determine the reference active tension and length.

All data were normalized to tension recordings obtained at $\mathrm{L}_{\text {ref }}$ based on prior studies showing reproducibility of this method in DSM $(8,9)$, accounting for variability in strip weight between DSM samples. Strips were equilibrated in KHB at slack length for 15 minutes. After a steady state was achieved, the passive force $\left(F_{p}\right)$ was recorded. The buffer was exchanged to high-potassium $\mathrm{KHB}(110.0 \mathrm{mM} \mathrm{KCl}$ and $8.7 \mathrm{mM} \mathrm{NaCl}$ ) for $30 \mathrm{sec}$ to induce muscle contraction and peak force was recorded. Active force $\left(F_{a}\right)$ at this muscle length was calculated by subtracting steady state $F_{p}$ recorded prior to contraction from peak force. Tissues were then washed in standard $\mathrm{KHB}$ for $5 \mathrm{~min}$, and $\mathrm{F}_{\mathrm{p}}$ at this new length was recorded. The process was repeated to identify a parabolic length-active tension curve and length-preload 
curve that increased non-linearly with increasing muscle length. Using this method, reference active force $\left(\mathrm{F}_{\mathrm{a}, \mathrm{ref}}\right)$ and its corresponding muscle length $\left(\mathrm{L}_{\mathrm{ref}}\right)$ were calculated for use in subsequent protocols.

\section{DSM experimental protocol}

Following determination of $\mathrm{L}_{\text {ref }}$, tissues were re-equilibrated for 30 minutes at $90 \% \mathrm{~L}_{\text {ref }}$ and the measured force referred to as "preload". They were then contracted in highpotassium KHB for 30 seconds and the generated force referred to as "afterload" (Figure 1A). The strips were subsequently washed into a zero-glucose KHB bubbled with $100 \% \mathrm{~N}_{2}$ gas to produce starved and hypoxic conditions for 120 minutes. After this exposure, tissues were contracted again in a high-potassium zero-glucose KHB for $30 \mathrm{~s}$ and then washed in standard KHB. The strips were placed in the aerated $\left(95 \% / 5 \% \mathrm{O}_{2} / \mathrm{CO}_{2}\right.$ gas) $\mathrm{KHB}$ tissue bath for a $15 \mathrm{~min}$ recovery period and then contracted for a third time in high-potassium KHB. These durations were determined and optimized based on preliminary serial observational studies performed over 150 -min periods in mouse, rabbit, and pig DSM tissue to characterize the effects of starvation/hypoxia on tissue functionality (unpublished data). The force at 15-min during the recovery (Tp3) divided by force at point Tp1 was referred to as "preload recovery". Additional tissue strip experiments were conducted in parallel in $\mathrm{KHB}$ with exposure to atropine $(1 \mu \mathrm{M})$ during the 120-min period of starvation/hypoxia. All DMS strip experiments were run with parallel control baths using tissue derived from the same porcine bladder.

Adjunct experiments were performed using denuded human DSM tissue. These strips were obtained from adult bladders removed from patients undergoing cystectomy for indicated clinical conditions at our affiliated medical centers. Whole bladders were transported on ice immediately after resection to a surgical pathologist who examined the gross surgical specimen and excised a full-thickness section of "normal" (non-pathologic) supra-trigonal bladder suitable for procurement of DSM strips. Human DSM strips were then prepared and studied in the aforementioned manner.

\section{Isolated perfused working pig bladder setup and apparatus}

Whole bladder experimental protocols were performed using a previously established ex vivo perfusion system (7). Immediately following procurement, excess tissue was removed and each vesical artery was cannulated using catheters which were filled with ice-cold KHB and then secured. Branch arteries were suture-ligated, and the right ureter was cannulated with a catheter used to continuously measure intra-vesical pressure $\left(\mathrm{P}_{\text {ves }}\right)$, and the left ureter was suture-ligated. A 16 F Foley catheter was secured in the urethral stump and inflated. After the bladder was placed in a dish located within an incubator (Symphony, VWR, Radnoti, PA, USA), each catheter was attached to connectors embedded in the incubator door, bladder vesical volume was adjusted to $50 \mathrm{~mL}$, and the bladder was perfused with warm $\left(37^{\circ} \mathrm{C}\right) \mathrm{KHS}$ and equilibrated for 45 minutes. As previously described, a gravity-feed was used to establish a constant-pressure perfusion system in which bladder blood vessel resistance determines organ perfusate flow (7). This was continuously measured using an in-line ultrasonic flowmeter (IUF-1000, Radnoti LLC, Monrovia, CA, USA). The perfusate was bubbled with $95 \% / 5 \% \mathrm{O}_{2} / \mathrm{CO}_{2}$ and pumped from a water-jacketed $2 \mathrm{~L}$ reservoir (\#120142-2, Radnoti LLC, Monrovia, CA, USA) to a bubble trap (\#130149, Radnoti LLC, Monrovia, CA, USA) open to air, which induced a pressure gradient for flow of $105 \mathrm{mmHg}$. The bladder was also super-fused with KHS warmed to $37^{\circ} \mathrm{C}$. Bladder filling was accomplished by a rate-controlled syringe pump (PHD 2000 Programmable, Harvard Apparatus, Holliston, MA, USA), and bladder $\mathrm{P}_{\text {ves }}$ was continuously monitored by a pressure transducer (P75, Harvard Apparatus, Holliston, MA, USA) attached to a ureteric cannula. Tissue oxygenation was also measured using an $\mathrm{O}_{2}$ probe inserted into the detrusor muscle on the anterior bladder wall (Oxylite 4000E, Oxfort Optronix Ltd, Abingdon, UK).

\section{Whole bladder ischemia protocol}

Following cannulation and setup within the perfusion apparatus, the bladder was permitted to further equilibrate for 60 minutes. The bladder was then filled to a volume of $510 \mathrm{~mL}$ at a rate of $40 \mathrm{cc} / \mathrm{min}$ as previously described for the first of three consecutive experimental fill/void cycles. The first fill/void, termed "pre-ischemia", was conducted with normal perfusate flow. Following the filling phase, the bladder was allowed to undergo a 5-min period of "stressrelaxation" (10). An intravascular bolus of $10 \mu \mathrm{M}$ carbachol in KHB was administered for $45 \mathrm{~s}$ with the urethral closed in order to elicit a 10-min "isovolumetric contraction (IVC)" (Figure 1B). The closed urethral catheter was subsequently opened, and the bladder was drained for a 5-min "voiding" period. Voided volume and post-void residual volume were 


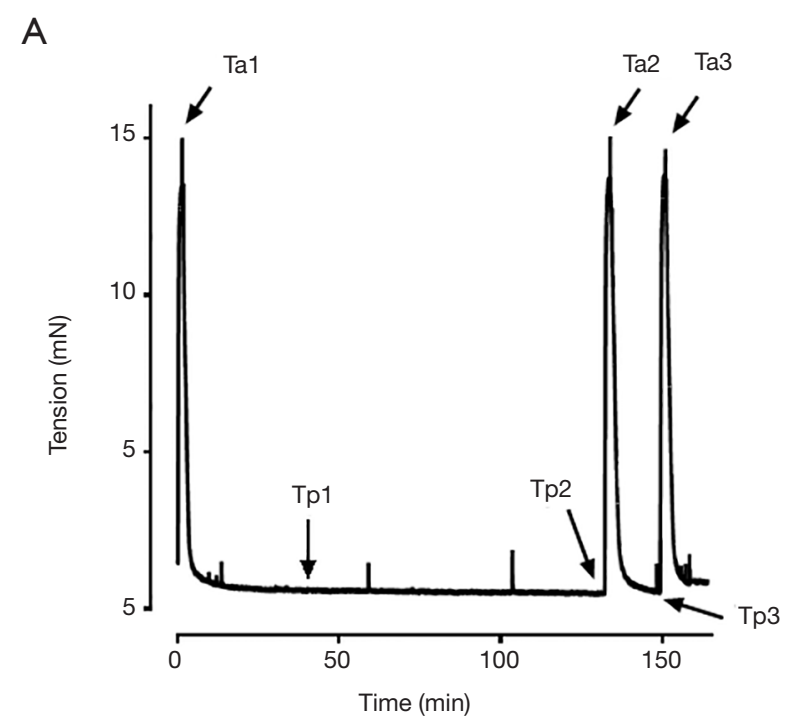

B

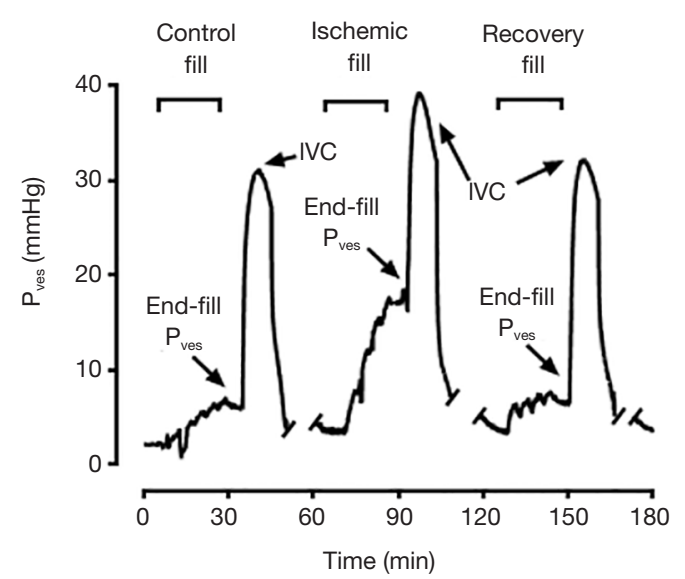

Figure 1 Diagrammatic representations of experimental protocols. (A) Representative tracings of pig DSM strip protocol in fed/oxygenated control strips. Contractile force or "afterload tension" was measured initially before intervention (Ta1), following $120 \mathrm{~min}$ (Ta2), and again after $15 \mathrm{~min}$ and (Ta3). Resting force or "preload tension" was measured at $45 \mathrm{~min}$ (Tp1), $120 \mathrm{~min}$ ( $\mathrm{Tp} 2$ ), and $15 \mathrm{~min}(\mathrm{Tp} 3)$; (B) example tracing of the pressure changes in the isolated perfused working whole pig bladder model during three consecutive cycles of filling and "voiding" during and after carbachol-stimulated IVC. Filling during the second cycle was performed under ischemic conditions, followed by a third recovery fill/void. DSM, detrusor smooth muscle; IVC, isovolumetric contraction; $\mathrm{P}_{\mathrm{ves}}$, vesical pressure.

recorded, and any residual intra-vesical fluid was drained. The bladder was re-equilibrated for a period of 45 minutes before the initiation of the second "ischemic" fill/void cycle. This subsequent filling cycle was conducted under ischemic conditions, with the perfusate inflow turned off during both the period of bladder filling and the 5-min "stressrelaxation" period. The perfusate flow was resumed in order to facilitate the carbachol-induced IVC. Following a final 45-min re-equilibration period, a third "recovery" fill/void cycle was performed with normal perfusate flow. Intra$\mathrm{P}_{\text {ves }}$ were recorded continuously throughout the fill/void cycles. Afterload, defined as the peak force of carbacholinduced contraction with the bladder outlet obstructed, was measured for each fill/void cycle. Experiments involving exposure to ischemic conditions were compared to a separate control group which only underwent 3 fill/void cycles under perfused conditions.

\section{Data analysis}

The data were analyzed using Graph Pad Prism 6.0 software (GraphPad Software, Inc., La Jolla, CA, USA) and are presented as representative tracings and mean \pm standard error of the mean (SEM). Data were evaluated with Student's $t$-test or the analysis of variance (ANOVA) with Dunnett's or Tukey's post hoc tests, as appropriate. The null hypothesis was rejected at $\mathrm{P}<0.05$.

\section{Results \\ DSM strips}

The pig DSM strips ( $\mathrm{n}=7$ ) were exposed to starved, hypoxic conditions in a muscle bath for a total of 2 hours (Figure 2). After an initial 15-min period of stress-relaxation, the ischemic muscle strips demonstrated a significant increase in preload tension as measured from $\mathrm{Tp} 1$ to $\mathrm{Tp} 2$ when compared to the control $(2.07 \pm 0.37$ vs. $0.98 \pm 0.04$-fold change, $\mathrm{P}<0.05$, Figure $3 A, B)$.

When the starved, hypoxic DSM strips were contracted in the high-potassium buffer, the force of contraction, or afterload tension, decreased by $46 \%$ in the experimental group as compared to control $(0.55 \pm 0.06$ vs. $1.01 \pm 0.03$-fold change, $\mathrm{P}<0.05$, Figure $3 C$ ). Following a return to the standard oxygenated $\mathrm{KHB}$, the previously starved/hypoxic strips demonstrated a significant recovery of afterload tension with a return to $84 \%$ of the pre-experimental contractile force $(0.85 \pm 0.02$ vs. $1.04 \pm 0.03$-fold change, $\mathrm{P}<0.05$, Figure 3C).

When the DSM strips were exposed to atropine during 


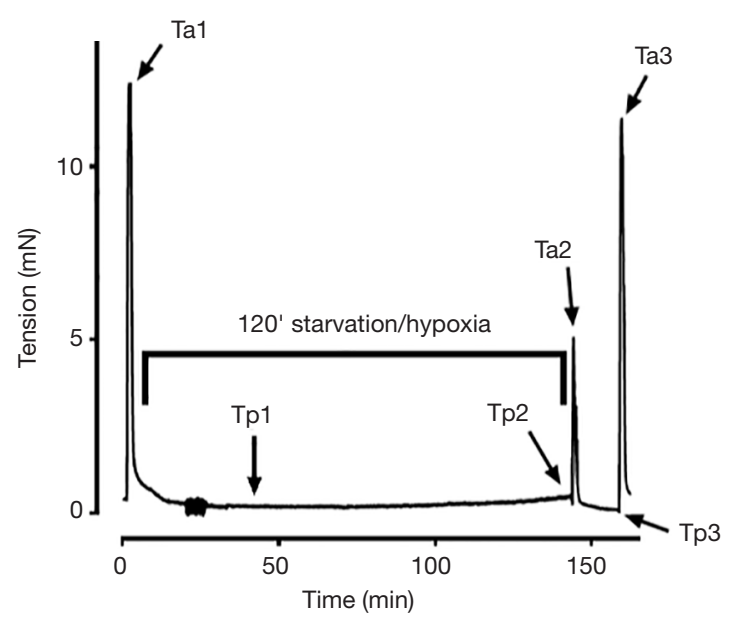

Figure 2 Representative tracings of pig DSM strip protocol in starved/hypoxic experimental strips. Contractile force or "afterload tension" was measured initially before intervention (Ta1), and again following $120 \mathrm{~min}$ starvation/hypoxia (Ta2). Strips recovered in a fed/oxygenated bath for $15 \mathrm{~min}$ and were then contracted a third time (Ta3). Resting force or "preload tension" was measured at $45 \mathrm{~min}$ (Tp1) and $120 \mathrm{~min}$ (Tp2) during starvation/hypoxia and 15 min after recovery (Tp3). DSM, detrusor smooth muscle.

the starved and hypoxic conditions $(n=4)$, the increase in resting preload tension was not seen, with no significant elevation in force over control strips $(0.89 \pm 0.04 \mathrm{vs}$. $0.96 \pm 0.08$-fold change, $\mathrm{P}=0.44$, Figure $3 D$ ).

When the strips underwent stress-relaxation for 15 minutes during the recovery period, the previously starved/hypoxic DSM strips maintained a degree of elevated preload tension; however, the difference failed to reach statistical significance compared to control $(2.6 \pm 1.0 \mathrm{vs}$. $0.94 \pm 0.2$-fold change, $\mathrm{P}=0.16, \mathrm{n}=7$ ). This increased preload tension was dampened by exposure to atropine $(1.62 \pm 0.65$ vs. $0.76 \pm 0.14$-fold change, $\mathrm{P}=0.32, \mathrm{n}=4$ ).

Additional experiments utilizing the pig DSM protocol were conducted in human DSM strips $(\mathrm{n}=2)$. Similar findings were demonstrated, wherein starved and hypoxic conditions led to an increase in resting preload tension $(\mathrm{Tp} 2 / \mathrm{Tp} 1)$. Again, this increased tension was not seen when experiment conducted in presence of atropine (data not shown).

\section{Isolated perfused working pig bladder apparatus}

Acute ischemic conditions were created in the isolated perfused working pig bladder $(n=3)$. When filled to $510 \mathrm{cc}$ under ischemic conditions, the increase in intra- $\mathrm{P}_{\mathrm{ves}}$ at the completion of filling was significantly higher than control $(15.69 \pm 1.11$ vs. $6.96 \pm 0.61 \mathrm{mmHg}, \mathrm{P}<0.05$, Figure $4 A)$.

When the bladder was re-perfused for a period of 20 minutes and then refilled to the predetermined capacity, complete recovery of normal bladder compliance was demonstrated in the next filling cycle, with a return to preischemia end-fill intra- $\mathrm{P}_{\text {ves }}$ (Figure $4 A$ ). Notably, there was no statistically significant difference between recovery endfill pressure and control $(7.34 \pm 1.22$ vs. $5.83 \pm 1.21 \mathrm{mmHg}$, $\mathrm{P}=0.43$, Figure $4 A$ ). The strength of the contractile force decreased under ischemic conditions; however, this was not statistically significant (Figure 4B).

When exposed to atropine immediately prior to ischemia, the increase in the end-fill intra- $\mathrm{P}_{\mathrm{ves}}$, or preload (Figure $5 A$ ), that was seen in the previous ischemic filling protocols was no longer noted (Figure $5 B$ ). The end-fill intra- $\mathrm{P}_{\text {ves }}$ during ischemic filling with atropine exposure was not significantly different from the pre-ischemic internal control fill cycle $(7.64 \pm 2.08$ vs. $8.28 \pm 1.67 \mathrm{mmHg}, \mathrm{n}=2$, Figure $4 B)$. Furthermore, the atropine exposure was associated with a near complete blockage of bladder contraction. Pressure during IVC decreased from $27.13 \pm 10.12 \mathrm{mmHg}$ in preischemic control down to $1.85 \pm 0.78 \mathrm{mmHg}$ with atropine exposure under ischemic conditions ( $\mathrm{n}=2$, Figure 5C).

In order to confirm that cessation of perfusate flow was leading to ischemic conditions in the IPWPB, tissue oxygenation levels were measured at varying perfusate flow rates. As seen in Figure 5D, decreasing tissue $\mathrm{pO}_{2}$ was found to correspond with decreasing perfusate flow rate $(\mathrm{n}=5$, $\mathrm{P}<0.05)$.

\section{Discussion}

The present study utilized a DSM organ bath preparation and isolated perfused whole working bladder system to evaluate the effect of acute tissue starvation, ischemia and hypoxia on the filling/storage and voiding phases. The acute effect on the filling/storage phase was evaluated with a 2-h period of tissue starvation and hypoxia in the pig DSM strips, leading to a significant rise in resting preload tension seen as an increase in DSM force (Figure 3B). This increase in resting preload tension was also observed in the whole bladder preparation, as demonstrated by the increased endfill intra- $\mathrm{P}_{\text {ves }}$ during ischemic filling and storage (Figure $4 A$ ).

The effect of tissue ischemia was further evaluated in the voiding phase where the contractile force in the pig DSM strips was decreased following exposure to starved and hypoxic conditions (Figure 3C). Notably however, a 
A

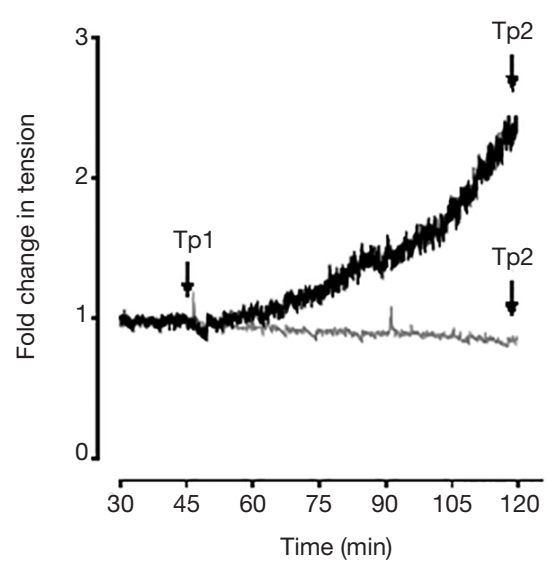

C

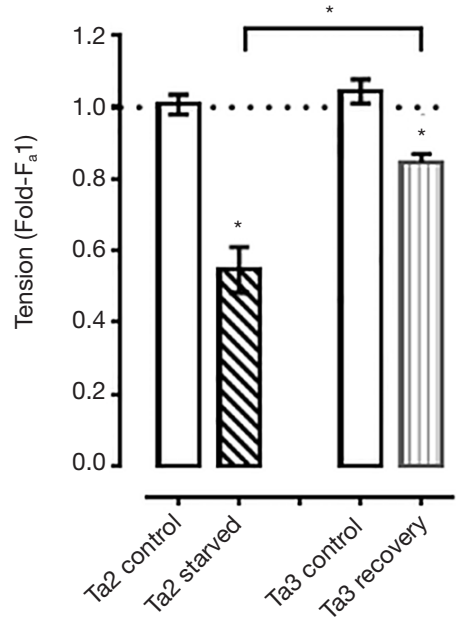

B

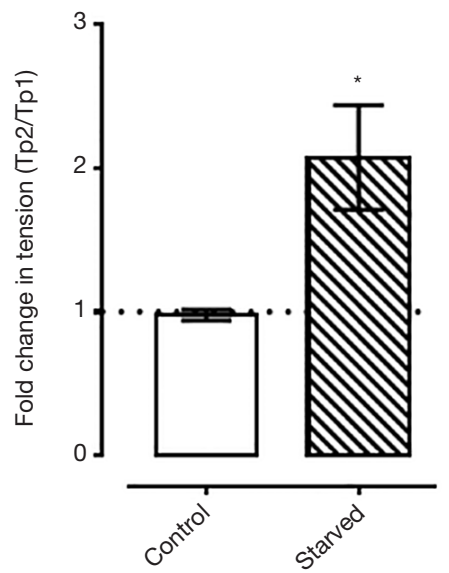

D

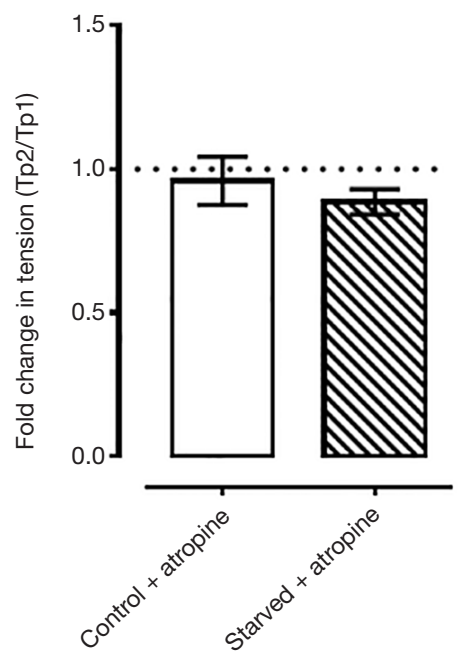

Figure 3 Pig DSM strips demonstrated a change in contractile force and resting preload force with starvation and hypoxia (n=7). (A) Example DSM strip fold change in resting preload tension over $120 \mathrm{~min}$ in a starved and hypoxic strip (black line) and a control strip (gray line); (B) fold change in contractile force over initial contraction (Ta1) after $120 \mathrm{~min}$ (Ta2) and following recovery (Ta3); (C) fold change in resting preload force at time-point $\mathrm{Tp} 2 / \mathrm{Tp} 1$; (D) fold change in resting preload tension with exposure to $1 \mu M$ atropine. Values shown are mean \pm SEM (error bars), ${ }^{*}, \mathrm{P}<0.05$. DSM, detrusor smooth muscle; SEM, standard error of the mean; $\mathrm{F}_{\mathrm{a}}$, active force.

short (15-min) period of re-feeding and re-oxygenation allowed for a return of contractile force to nearly $90 \%$ of pre-experimental control force, suggesting that the acute ischemic effects may be reversible. Mirroring the tissue recovery seen in the DSM protocol, the end-fill pressure in the whole bladder preparation returned to normal with re-perfusion (Figure 4A), while the strength of the IVC recovered similarly to the strength of pre-ischemia controls (Figure 4B).

In an effort to further delineate potential mechanisms for the changes seen with ischemia, the DSM and working perfused whole bladder preparation experimental protocols were performed with exposure to atropine in order to explore the effect of an anticholinergic drug on the detrusor function. When exposed to atropine, the increase in resting force during DSM tissue starvation/hypoxia was blocked (Figure 3D), suggesting that local acetylcholine release plays a role in the tissue response to these conditions. Similarly, the elevated end-fill pressure seen in the ischemic whole bladder model was also blocked by the atropine, supporting this observation (Figure 5C).

In a study of anesthetized dogs, Azadzoi et al. first 
A

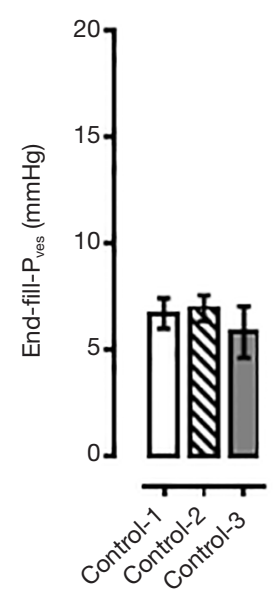

B

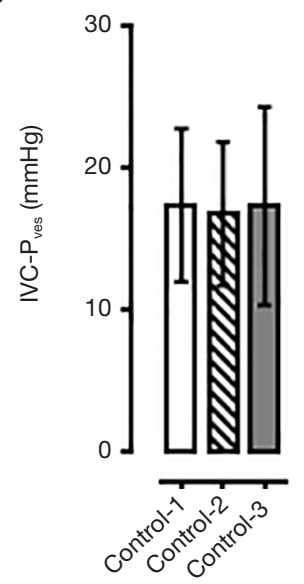

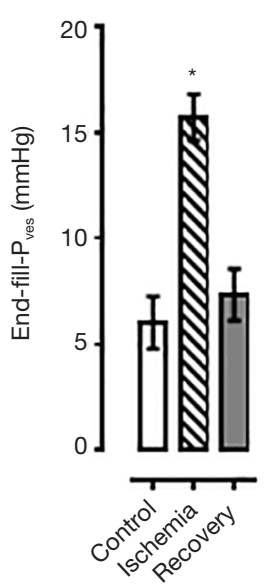

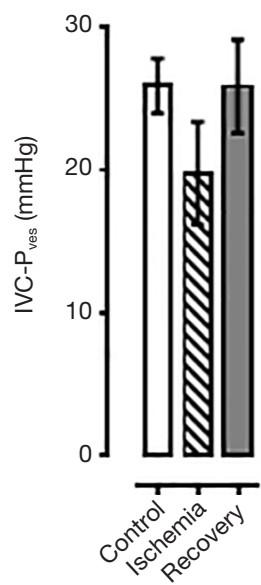

Figure 4 Pressure measured during filling in isolated perfused working whole pig bladder. (A) When the bladder was filled under ischemic conditions, the end-fill $\mathrm{P}_{\text {ves }}$ was significantly elevated over control $(n=3)$. The subsequent recovery filling cycle with normal perfusate flow (non-ischemic conditions) showed a return to baseline end-fill $\mathrm{P}_{\text {ves }}(\mathrm{n}=3)$; (B) the strength of the carbacholinduced contraction following immediate reperfusion was not significantly decreased over control $(\mathrm{n}=3)$. Values shown are mean \pm SEM (error bars), * $\mathrm{P}<0.05$. SEM, standard error of the mean; IVC, isovolumetric contraction; $\mathrm{P}_{\mathrm{ves}}$, vesical pressure.

demonstrated a decrease in bladder wall blood flow and tissue oxygenation during the bladder filling and contraction. The ischemia and hypoxia they found have been suggested to play a role in bladder pathology (11). Subsequent studies have shown that during a normal fill/void cycle, the bladder experiences temporary reversible ischemia (12). Additionally, acute urinary retention leads to a temporary ischemia, but flow returns to basal levels

A

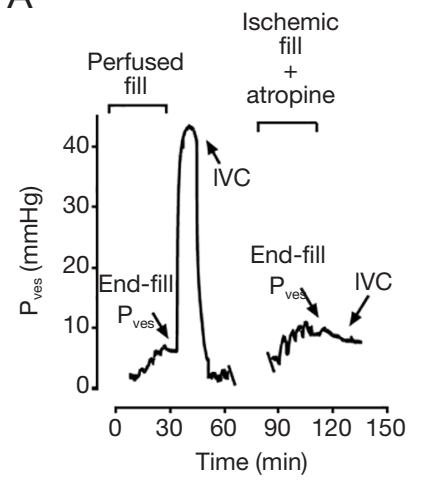

B

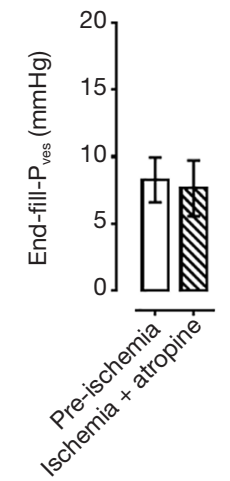

C
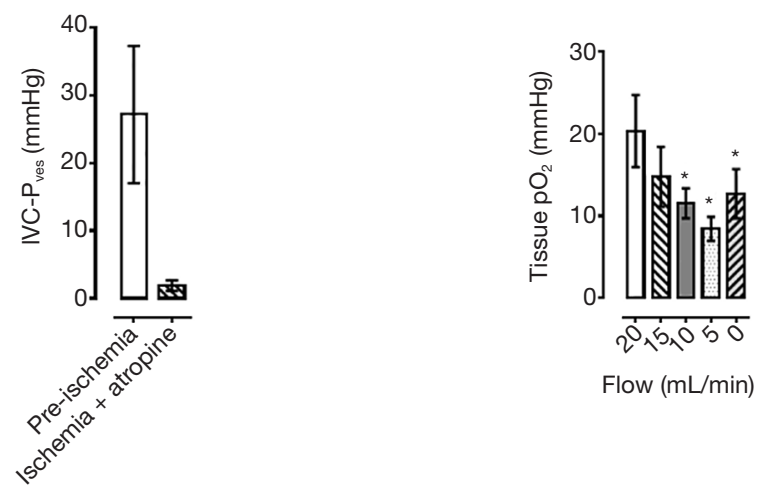

Figure 5 Isolated perfused working whole pig bladder experiments performed in presence of atropine. (A) Example tracing of pressure changes with perfused filling and contraction followed by ischemic fill and contraction. Bladder was treated with atropine immediately prior to ischemia; (B) end-fill $\mathrm{P}_{\text {ves }}$ measured following exposure to atropine shows no significant difference over control $(n=2)$, while carbachol-stimulated isovolumetric bladder contraction (C) was completely blocked; (D) detrusor tissue $\mathrm{pO} 2$ measured at decreasing arterial perfusate flow rates $(\mathrm{n}=5)$. Values shown are mean \pm SEM (error bars), *, $\mathrm{P}<0.05$. SEM, standard error of the mean; IVC, isovolumetric contraction; $\mathrm{P}_{\mathrm{ves}}$, vesical pressure.

following drainage (13). However, despite these regular ischemic episodes, bladders are able to return to and maintain normal functionality. This has been attributed to protective mechanisms that prevent long-term pathologic damage from the transient ischemia seen in normal fill/ void cycles $(14,15)$. Despite these protective mechanisms, when the ischemia occurs over longer periods of time deleterious effects are noted. Chronic ischemia has been closely associated with pathologic progression to detrusor 
overactivity, and eventually detrusor underactivity $(1,16)$. While clear pathophysiologic mechanisms have yet to be elucidated, preclinical studies have demonstrated changes such upregulation in oxidative stress genes and increased muscarinic receptor activity, which have been associated with bladder hyperactivity when exposed to moderate ischemia (17-19). In the setting of severe or chronic ischemia, decreased functionality in the form of bladder underactivity and decreased urodynamic micturition frequency, have been identified (6). However, it should be noted that several of these studies have yielded conflicting findings (1). Prior to the present study, little was known about the effects of a transient ischemic "attack" on the bladder.

Previous studies using this whole bladder perfusion system have elucidated a potential mechanism for vascular autoregulation in the low-flow state (7). Anele et al. showed that while flows above $5 \mathrm{~mL} / \mathrm{min}$ correlated with perfusion pressures of $>100 \mathrm{mmHg}$, there was a deviation from linearity in the pressure-flow relationship below $5 \mathrm{~mL} / \mathrm{min}$. This suggests that the autoregulatory mechanisms are activated at "very low flow" or complete ischemia as demonstrated by a drop in vascular resistance at this low flow rate (2). These mechanisms are likely involved in the increased intravesical storage pressure response to ischemia and hypoxia/starved conditions. The contraction of the detrusor muscle seen with ischemic filling and storage is atropinesensitive, suggesting that this increased tone is secondary to a local release of acetylcholine. The source of the acetylcholine release is likely from neurons or DSM cells given that the DSM strips were denuded. Furthermore, the ex vivo whole bladder studies were conducted without an intact autonomic nervous system to contribute to cholinergic input.

We hypothesize that prolonged ischemic episodes cause a local release of acetylcholine from bladder postganglionic parasympathetic nerves and consequently results in bladder overactivity. This release is insufficient to cause a "voiding-like" contraction but is enough to reduce bladder compliance. This may explain the efficacy of antimuscarinic drugs in the treatment certain patients with symptoms of overactive bladder. Previous studies have shown a potentially protective effect of anticholinergic (and more specifically antimuscarinic) drugs on prevention of ischemic bladder changes (20). The similar findings in the human DSM strips also suggest potential application in the clinical setting.

While the present study does demonstrate remarkable properties of the porcine bladder, there are a number of notable limitations. While one may speculate on the mechanism behind the increased detrusor tone with ischemia based on the observed blocking effect of atropine, molecular studies are needed to further evaluate and confirm the findings. Measurements of acetylcholine and adenosine triphosphate (ATP) levels in future studies may be warranted given the established relationship demonstrated in other similar animal models $(21,22)$. The scope of the data is also limited by the relatively low number of bladder and detrusor specimens, but the consistency of the results provides reassurance that findings are valid. Furthermore, preliminary studies utilizing human DSM strips support the conclusions; however, further studies are needed. As an isolated organ, the perfused working whole bladder model lacks autonomic innervation which limits the ability to evaluate effects of extrinsic neural input to the bladder, but this also allows assessment of intrinsic local neuronal effects. Finally, there are notable contributions by the urothelium which were not evaluated in this present study but likely play a fundamental mediating role (23).

\section{Conclusions}

Transient ischemia/hypoxia leads to an acute increase in tone in both DSM strips and a perfused working whole bladder model. Importantly, the effect is reversible with re-perfusion and may be blocked with anticholinergics, suggesting a relationship between acute ischemia and increased local acetylcholine release.

\section{Acknowledgments}

We would also like to extend a special thanks to David Burks of Burks Farm, Providence Forge, VA 23140, USA; and Mike Silver of Silver Ridge Farm, Fredericksburg, VA 22405, USA. They were generous and hospitable in donating pig bladders and in providing accommodations to prepare the bladders on-site. In addition, Sydney Roberts and Ryan Musselman provided valuable assistance with the experiments.

\section{Footnote}

Conflicts of Interest: The authors have no conflicts of interest to declare.

Ethical Statement: The authors are accountable for all aspects of the work in ensuring that questions related to the accuracy or integrity of any part of the work are 
appropriately investigated and resolved. All experiments involving human tissue received approval from the Virginia Commonwealth University institutional review board (HM 20009688) and informed consent was obtained from patients prior to their use.

\section{References}

1. Andersson KE, Boedtkjer DB, Forman A. The link between vascular dysfunction, bladder ischemia, and aging bladder dysfunction. Ther Adv Urol 2017;9:11-27.

2. Anele UA, Ratz PH, Colhoun AF, et al. Potential vascular mechanisms in an ex vivo functional pig bladder model. Neurourol Urodyn 2018;37:2425-33.

3. Thurmond P, Yang JH, Azadzoi KM. LUTS in pelvic ischemia: a new concept in voiding dysfunction. Am J Physiol Renal Physiol 2016;310:F738-43.

4. Lin WY, Andersson KE, Lin CL, et al. Association of lower urinary tract syndrome with peripheral arterial occlusive disease. PLoS One 2017;12:e0170288.

5. Zhao Z, Azad R, Yang JH, et al. Progressive changes in detrusor function and micturition patterns with chronic bladder ischemia. Investig Clin Urol 2016;57:249-59.

6. Azadzoi KM, Tarcan T, Siroky MB, et al. Atherosclerosisinduced chronic ischemia causes bladder fibrosis and noncompliance in the rabbit. J Urol 1999;161:1626-35.

7. Vince R, Tracey A, Deebel NA, et al. Effects of vesical and perfusion pressure on perfusate flow, and flow on vesical pressure, in the isolated perfused working pig bladder reveal a potential mechanism for the regulation of detrusor compliance. Neurourol Urodyn 2018;37:642-9.

8. Speich JE, Almasri AM, Bhatia H, et al. Adaptation of the length-active tension relationship in rabbit detrusor. Am J Physiol Renal Physiol 2009;297:F1119-28.

9. Almasri AM, Ratz PH, Bhatia H, et al. Rhythmic contraction generates adjustable passive stiffness in rabbit detrusor. J Appl Physiol (1985) 2010;108:544-53.

10. Speich JE, Quintero K, Dosier C, et al. A mechanical model for adjustable passive stiffness in rabbit detrusor. J Appl Physiol (1985) 2006;101:1189-98.

11. Azadzoi KM, Pontari M, Vlachiotis J, et al. Canine bladder blood flow and oxygenation: changes induced by filling, contraction and outlet obstruction. J Urol 1996;155:1459-65.

12. Greenland JE, Brading AF. Urinary bladder blood flow changes during the micturition cycle in a conscious pig model. J Urol 1996;156:1858-61.

13. Shimizu S, Saito M, Kinoshita Y, et al. Acute urinary retention and subsequent catheterization cause lipid peroxidation and oxidative DNA damage in the bladder: preventive effect of edaravone, a free-radical scavenger. BJU Int 2009;104:713-7.

14. Bratslavsky G, Kogan BA, Matsumoto S, et al. Reperfusion injury of the rat bladder is worse than ischemia. J Urol 2003;170:2086-90.

15. Madersbacher H, Cardozo L, Chapple C, et al. What are the causes and consequences of bladder overdistension? ICI-RS 2011. Neurourol Urodyn 2012;31:317-21.

16. Chancellor MB. The overactive bladder progression to underactive bladder hypothesis. Int Urol Nephrol 2014;46 Suppl 1:S23-7.

17. Azadzoi KM, Radisavljevic ZM, Golabek T, et al. Oxidative modification of mitochondrial integrity and nerve fiber density in the ischemic overactive bladder. J Urol 2010;183:362-9.

18. Azadzoi KM, Tarcan T, Kozlowski R, et al. Overactivity and structural changes in the chronically ischemic bladder. J Urol 1999;162:1768-78.

19. Azadzoi KM, Yalla SV, Siroky MB. Oxidative stress and neurodegeneration in the ischemic overactive bladder. J Urol 2007;178:710-5.

20. Scheepe JR, de Jong BW, Wolffenbuttel KP, et al. The effect of oxybutynin on structural changes of the obstructed guinea pig bladder. J Urol 2007;178:1807-12.

21. McCarthy CJ, Marangos C, Fry CH, et al. ATP transients accompany spontaneous contractions in isolated guinea-pig detrusor smooth muscle. Exp Physiol 2019;104:1717-25.

22. Stenqvist J, Winder M, Carlsson T, et al. Urothelial acetylcholine involvement in ATP-induced contractile responses of the rat urinary bladder. Eur J Pharmacol 2017;809:253-60.

23. Guan NN, Gustafsson LE, Svennersten K. Inhibitory effects of urothelium-related factors. Basic Clin Pharmacol Toxicol 2017;121:220-4.

Cite this article as: Tracey AT, Anele UA, Vince RA, Speich JE, Klausner AP, Ratz PH. Bladder attack: transient bladder ischemia leads to a reversible decrease in detrusor compliance. Transl Androl Urol 2019;8(6):703-711. doi: 10.21037/ tau.2019.11.12 